\title{
Numerical solution of Sturm-Liouville problems via Fer streamers
}

\author{
Alberto Gil C. P. Ramos · Arieh Iserles
}

the date of receipt and acceptance should be inserted later

\begin{abstract}
We address the numerical challenge of solving regular Sturm-Liouville problems in Liouville's normal form, with a continuous and piecewise analytic potential and self-adjoint separated boundary conditions. The novelty of our approach, which is based on a non-standard truncation of Fer expansions, which we call 'Fer streamers', lies in the construction of a new numerical method, which, $i$ ) does not impose any restriction on the step size for eigenvalues which are greater than or equal to the minimum of the potential, $i i$ ) requires only a mild restriction on the step size for the remaining finite number of eigenvalues, iii) can attain any convergence rate, which grows exponentially with the number of terms, and is uniform for every eigenvalue, and, $i v$ ) lends itself to a clear understanding of the manner in which the potential affects the local and global errors. We provide our numerical method with its analytical underpinning, but emphasize that it is at an early stage of development and that much remains to be done. In particular, we comment on our investigation of efficient discretization schemes for the integrals which arise in Fer streamers.
\end{abstract}

Keywords Numerical method · Regular Sturm-Liouville problems · Liouville's normal form $\cdot$ Continuous and piecewise analytic potential $\cdot$ Self-adjoint separated boundary conditions $\cdot$ Fer expansions $\cdot$ Fer streamers

Mathematics Subject Classification (2010) 65L15 $65 \mathrm{~L} 10 \cdot 65 \mathrm{~L} 70 \cdot 34 \mathrm{C} 40$

Alberto Gil C. P. Ramos

Cambridge Centre for Analysis, University of Cambridge, United Kingdom

E-mail: A.G.C.P.Ramos@maths.cam.ac.uk

Arieh Iserles

Department of Applied Mathematics and Theoretical Physics, University of Cambridge, United Kingdom E-mail: A.Iserles@damtp.cam.ac.uk 


\section{Introduction}

We address the numerical challenge of solving regular Sturm-Liouville problems in Liouville's normal form with a continuous and piecewise analytic potential

$$
\begin{gathered}
-y_{\lambda}^{\prime \prime}(t)+q(t) y_{\lambda}(t)=\lambda y_{\lambda}(t), \quad t \in[a, b], \quad a, b \in \mathbb{R}, \quad \lambda \in \mathbb{R}, \\
q \in C^{0}\left([a, b],\left[q_{\min }, q_{\max }\right]\right) \text { is piecewise analytic, } \quad y_{\lambda} \in C^{2}([a, b], \mathbb{R}),
\end{gathered}
$$

and self-adjoint separated boundary conditions

$$
\begin{array}{ccc}
\alpha_{1} y_{\lambda}(a)+\alpha_{2} y_{\lambda}^{\prime}(a)=0, & \alpha_{1}, \alpha_{2} \in \mathbb{R}, & \alpha_{1}^{2}+\alpha_{2}^{2}>0, \\
\beta_{1} y_{\lambda}(b)+\beta_{2} y_{\lambda}^{\prime}(b)=0, & \beta_{1}, \beta_{2} \in \mathbb{R}, & \beta_{1}^{2}+\beta_{2}^{2}>0,
\end{array}
$$

where $a, b, q, q_{\min }, q_{\max }, \alpha_{1}, \alpha_{2}, \beta_{1}$ and $\beta_{2}$ are known, and the eigenvalue and eigenfunction pairs $\left(\lambda, y_{\lambda}\right)$ are unknown (Everitt, 2005).

Numerical methods for these problems, as well as for general Sturm-Liouville problems, which, occasionally, can also be placed in Liouville's normal form via Liouville's transformation (Everitt, 2005, p. 279-280), are particularly important in physics, chemistry and applied mathematics, e.g., in fluid flow, Schrödinger spectra, nuclear magnetic resonance imaging, etc (Iserles, Munthe-Kaas, Nørsett and Zanna 2000, p. 333-337).

It has been known for many years that regular Sturm-Liouville problems in Liouville's normal form, with a continuous (not necessarily piecewise analytic) potential and self-adjoint separated boundary conditions (1)-(2), possess a unique countable family of solutions $\left\{\left(\lambda_{j}, y_{\lambda_{j}}\right): j \in \mathbb{Z}_{0}^{+}\right.$and $\left.\left\|y_{\lambda_{j}}\right\|_{L^{2}([a, b], \mathbb{R})}=1\right\}$, which can be shown to satisfy,

$$
\begin{gathered}
\lambda_{j}<\lambda_{j+1} \text { and } \lim _{j \rightarrow+\infty} \lambda_{j}=+\infty, \\
y_{\lambda_{j}} \text { has exactly } j \text { zeros in }(a, b), \\
\left\{y_{\lambda_{j}}\right\}_{j \in \mathbb{Z}_{0}^{+}} \text {is an orthonormal basis of } L^{2}([a, b], \mathbb{R}) .
\end{gathered}
$$

Notwithstanding this result, the fact remained that, although there existed fairly robust methods to compute $\left(\lambda_{j}, y_{\lambda_{j}}\right)$ for small $j$, it was infeasible, in practice, to compute eigenvalue and eigenfunction pairs whenever $j \gg 1$. In particular, every numerical method designed for Sturm-Liouville problems, was either: $i$ ) unable to compute arbitrarily large eigenvalues because of severe restrictions on the step size, or, $i i$ ) able to compute arbitrarily large eigenvalues, but unable to provide a fast convergence rate in terms of the step size (Pryce, 1993).

In recent years, this state of affairs has began to change with the contributions of (Moan, 1998), (Iserles et al. 2000, p. 116-120), (Ixaru, 2000), (Ledoux, Daele and van den Berghe, 2010), etc.

Moan's (1998) work, is based on four ideas: $i$ ) in formulating the Sturm-Liouville problem (1)-2) in the Lie-group

$$
\operatorname{SL}(2, \mathbb{R}):=\left\{\left[\begin{array}{ll}
a & b \\
c & d
\end{array}\right]: a, b, c, d \in \mathbb{R} \text { and } a d-b c=1\right\}
$$


of two-by-two real matrices with determinant one, $i$ ) in approximating the solution in $\operatorname{SL}(2, \mathbb{R})$ with the use of the Lie-algebra

$$
\mathfrak{s l}(2, \mathbb{R}):=\left\{\left[\begin{array}{ll}
a & b \\
c & -a
\end{array}\right]: a, b, c \in \mathbb{R}\right\}
$$

of two-by-two real matrices with zero trace and Magnus expansions, by calling upon (Iserles and Nørsett, 1999), iii) in discretizing Magnus expansions, with discretization schemes put forth in (Iserles and Nørsett, 1999), and, $i v$ ) in a clever summation of the discretized terms in order to avoid some of the issues that arise with large eigenvalues. In particular, in his work, Moan (1998) established a numerical method with global order 4 which is able to approximate uniformly any eigenvalue within the bounded interval $h^{2}|\lambda| \leq 1$, where $h$ denotes the step size.

Following Moan's (1998) work, Iserles et al.'s (2000) suggested a slightly different, but game-changing, approach: in short, switch the order of discretization and clever summation. This new idea, coined 'Magnus streamers', opened the door to truly fast computations of Magnus series in low-dimensional Lie algebras, making it an important contribution to the solution of matrix Lie-group linear differential equations. Unfortunately, when applied to the formulation of the Sturm-Liouville problem (1)-(2) in $\operatorname{SL}(2, \mathbb{R})$, the uniform approximation of Magnus streamers turn out to be prohibitively complex: there is nothing wrong with the summation, except that it is difficult to track the manner in which the magnitude of the eigenvalue influences the local and global error estimates.

In this paper, we apply the idea from (Iserles et al. 2000) to Fer expansions' instead of Magnus expansions. The result is remarkable: by calling upon Fer expansions radius of convergence and recursive nature, it turns out that, unlike Magnus streamers, 'Fer streamers' lend themselves to uniform approximation of every eigenvalue and eigenfunction pair and exponentially growing order with increasing number of terms, making them a perfect tool in our endeavor!

Indeed, based on this new concept called Fer streamers, we propose a new numerical method, which, $i$ ) does not impose any restriction on the step size for eigenvalues which are greater than or equal to the minimum of the potential, $i i$ ) requires only a mild restriction on the step size for the remaining finite number of eigenvalues, iii) can attain any convergence rate, which grows exponentially with the number of terms, and is uniform for every eigenvalue, and, $i v$ ) lends itself to a clear understanding of the manner in which the potential affects the local and global errors.

It is important to emphasize that the piecewise perturbation methods in (Ixaru 2000) and the modified Magnus methods in (Ledoux et al. 2010), also feature a numerical mesh which is not severely impeded by the magnitude of the eigenvalues but lead to asymptotic estimates.

Our main contribution to the state-of-the-art is three-fold. Firstly, the novelty of our approach. We produce a new numerical method with a numerical mesh which is virtually independent of the size of the eigenvalues and leads to uniform estimates, by introducing a new set of ideas based on Fer streamers. Secondly, the order in our numerical method grows exponentially with the number of terms, as opposed to the linear growth in (Ixaru, 2000) and (Ledoux et al., 2010). Thirdly, unlike (Ixaru, 2000) 
and (Ledoux et al., 2010), we provide a clear understanding of how the potential affects our local and global errors.

Assumption 1 The numerical mesh

$$
\begin{gathered}
m \in \mathbb{Z}^{+}, \\
c_{0}:=a<c_{1}<\cdots<c_{m-1}<c_{m}:=b, \\
h_{\min }:=\min _{k \in\{0,1, \ldots, m-1\}}\left\{c_{k+1}-c_{k}\right\}, \\
h_{\max }:=\max _{k \in\{0,1, \ldots, m-1\}}\left\{c_{k+1}-c_{k}\right\},
\end{gathered}
$$

is such that

$$
\begin{aligned}
& \left.\begin{array}{r}
k \in\{0,1, \ldots, m-1\} \\
t \in\left(c_{k}, c_{k+1}\right)
\end{array}\right\} \quad \Longrightarrow \quad q(t)=\sum_{j=0}^{\infty} \frac{q^{(j)}\left(c_{k}^{+}\right)}{j !}\left(t-c_{k}\right)^{j}, \\
& \lambda \geq q_{\min } \quad \Longrightarrow \quad h_{\max } \leq \frac{1}{\sqrt{q_{\max }-q_{\min }}}, \\
& \lambda<q_{\min } \quad \Longrightarrow \quad h_{\max } \leq \frac{1}{\sqrt{q_{\max }-\lambda}}, \\
& \frac{h_{\max }}{h_{\min }} \leq 2 \text { (this constant can be increased). }
\end{aligned}
$$

Remark 1 There exist Sturm-Liouville problems (1)-2) where (5) does not need to be considered because there do not exist eigenvalues which are less than the minimum of the potential. For example, if the boundary conditions (2) are such that

$$
-y_{\lambda}^{\prime}(b) y_{\lambda}(b)+y_{\lambda}^{\prime}(a) y_{\lambda}(a) \geq 0
$$

then

$$
\begin{aligned}
& -y_{\lambda}^{\prime \prime}(t)+q(t) y_{\lambda}(t)=\lambda y_{\lambda}(t) \Rightarrow \\
& \Rightarrow \int_{a}^{b}\left(-y_{\lambda}^{\prime \prime}(t) y_{\lambda}(t)+q(t)\left(y_{\lambda}(t)\right)^{2}\right) d t=\lambda \int_{a}^{b}\left(y_{\lambda}(t)\right)^{2} d t \\
& \Leftrightarrow \lambda=\frac{-y_{\lambda}^{\prime}(b) y_{\lambda}(b)+y_{\lambda}^{\prime}(a) y_{\lambda}(a)+\int_{a}^{b}\left(\left(y_{\lambda}^{\prime}(t)\right)^{2}+q(t)\left(y_{\lambda}(t)\right)^{2}\right) d t}{\int_{a}^{b}\left(y_{\lambda}(t)\right)^{2} d t} \\
& \Rightarrow \lambda \geq q_{\min } .
\end{aligned}
$$

Important examples of boundary conditions (2) that satisfy (7) include zero Dirichlet

$$
\alpha_{1} \neq 0, \quad \beta_{1} \neq 0, \quad \alpha_{2}=\beta_{2}=0, \quad y_{\lambda}(a)=y_{\lambda}(b)=0
$$

and zero Neumann

$$
\alpha_{1}=\beta_{1}=0, \quad \alpha_{2} \neq 0, \quad \beta_{2} \neq 0, \quad y_{\lambda}^{\prime}(a)=y_{\lambda}^{\prime}(b)=0
$$


boundary conditions, but 8 is not true in general. As an example, let

$$
a=0, \quad b=\pi, \quad(\forall t \in[0, \pi], q(t)=0), \quad \alpha_{1}=\alpha_{2} \neq 0, \quad \beta_{1}=\beta_{2} \neq 0
$$

and consider the regular Sturm-Liouville problem in Liouville's normal form with self-adjoint separated boundary conditions

$$
-y_{\lambda}^{\prime \prime}(t)=\lambda y_{\lambda}(t), \quad t \in[0, \pi], \quad y_{\lambda}(0)+y_{\lambda}^{\prime}(0)=0, \quad y_{\lambda}(\pi)+y_{\lambda}^{\prime}(\pi)=0
$$

with eigenvalues and eigenfunctions (normalized so that $\int_{0}^{\pi}\left(y_{\lambda}(t)\right)^{2} d t=1$ ) given in closed-form by

$$
\begin{gathered}
\lambda_{j}= \begin{cases}-1 & \Leftarrow j=0, \\
j^{2} & \Leftarrow j \in \mathbb{Z}^{+},\end{cases} \\
y_{\lambda_{j}}(t)= \begin{cases}\frac{e^{-t}}{e^{-\frac{\pi}{2}} \sqrt{\sinh (\pi)}} & \Leftarrow j=0, \\
\frac{j \cos (j t)-\sin (j t)}{\sqrt{\frac{\pi}{2}} \sqrt{j^{2}+1}} & \Leftarrow j \in \mathbb{Z}^{+} .\end{cases}
\end{gathered}
$$

In this example, (8) does not hold true because the negative eigenvalue, $\lambda_{0}=-1$, is strictly smaller than the minimum of the potential, $q_{\min }=0$.

Our approach consists of a three-step procedure, which is based on Assumption 11. Firstly, we divide (4) and (5) into two pieces

$$
\lambda \in\left[q_{\max }-h_{\max }^{-2}, q_{\max }+h_{\max }^{-2}\right] \cup\left[q_{\max }+h_{\max }^{-2},+\infty\right)
$$

and we approximate the solution of

$$
\mathbf{Y}_{\lambda}^{\prime}(t)=\left[\begin{array}{cr}
0 & 1 \\
q(t)-\lambda & 0
\end{array}\right] \mathbf{Y}_{\lambda}(t), \quad t \in[a, b], \quad a, b \in \mathbb{R}, \quad \lambda \in \mathbb{R},
$$

$q \in C^{0}\left([a, b],\left[q_{\min }, q_{\max }\right]\right)$ is piecewise analytic, $\quad \mathbf{Y}_{\lambda}:[a, b] \rightarrow \operatorname{SL}(2, \mathbb{R})$,

with initial condition

$$
\mathbf{Y}_{\lambda}(a)=\left[\begin{array}{ll}
1 & 0 \\
0 & 1
\end{array}\right]
$$

in the two uniform regimes

$$
\begin{gathered}
h_{\max } \rightarrow 0^{+}, \text {uniformly w.r.t. }\left\{\begin{array}{l}
k \in\{0,1, \ldots, m-1\}, t \in\left[c_{k}, c_{k+1}\right], \\
\lambda \in\left[q_{\max }-h_{\max }^{-2}, q_{\max }+h_{\max }^{-2}\right],
\end{array}\right. \\
h_{\max } \rightarrow 0^{+}, \text {uniformly w.r.t. }\left\{\begin{array}{l}
k \in\{0,1, \ldots, m-1\}, t \in\left[c_{k}, c_{k+1}\right], \\
\lambda \in\left[q_{\max }+h_{\max }^{-2},+\infty\right) .
\end{array}\right.
\end{gathered}
$$

Our main ideas lie precisely in the development of these uniform expansions. We proceed by recalling Fer expansions and observing that the error in the standard truncation of Fer expansions deteriorates with increasing values of $\lambda$. This, at first glance, suggests that Fer expansions are not a useful tool to increase the step size in the presence of large eigenvalues, but nothing could be further from the truth! Indeed, it is 
possible to truncate Fer expansions in an alternative manner, with what we call Fer streamers, which, to all intents and purposes, do not impede the step size and yield error estimates with exponentially growing order with increasing number of terms which also single out the role of the potential!

Remark 2 There is a geometric feature intrinsic to (9)-(10) that should not go unnoticed: as indicated above, it is true that (c.f., (Iserles et al., 2000, p. 215-252))

$$
\mathbf{Y}_{\lambda}([a, b]) \subseteq \operatorname{SL}(2, \mathbb{R})
$$

The reader should be aware that the uniform expansions that appear in this paper preserve this geometrical feature.

Secondly, we approximate the eigenvalues $\lambda$, either via a shooting method (Pryce 1993, p. 88-116) based on

$$
\begin{aligned}
{\left[\begin{array}{cc}
\alpha_{1} & \alpha_{2} \\
0 & 0
\end{array}\right]\left[\begin{array}{l}
y_{\lambda}(a) \\
y_{\lambda}^{\prime}(a)
\end{array}\right]+\left[\begin{array}{cc}
0 & 0 \\
\beta_{1} & \beta_{2}
\end{array}\right]\left[\begin{array}{l}
y_{\lambda}(b) \\
y_{\lambda}^{\prime}(b)
\end{array}\right] } & =\left[\begin{array}{l}
0 \\
0
\end{array}\right], \\
{\left[\begin{array}{l}
y_{\lambda}(b) \\
y_{\lambda}^{\prime}(b)
\end{array}\right] } & =\mathbf{Y}_{\lambda}(b)\left[\begin{array}{l}
y_{\lambda}(a) \\
y_{\lambda}^{\prime}(a)
\end{array}\right],
\end{aligned}
$$

or via the transcendental characterization (Zettl, 2005, p. 43-47) given by

$$
\left\{\lambda_{j}\right\}_{j \in \mathbb{Z}_{0}^{+}}=\left\{\lambda \in \mathbb{R}: \operatorname{det}\left(\left[\begin{array}{cc}
\alpha_{1} & \alpha_{2} \\
0 & 0
\end{array}\right]+\left[\begin{array}{cc}
0 & 0 \\
\beta_{1} & \beta_{2}
\end{array}\right] \mathbf{Y}_{\lambda}(b)\right)=0\right\},
$$

which relate (1)-(2) and (9)-(10), by approximating $\mathbf{Y}_{\lambda}(b)$ with Fer streamers, and solving the resulting equation with the use of a root-finding algorithm.

Thirdly, having approximated the eigenvalues, we continue by estimating the corresponding eigenfunctions $y_{\lambda}$ using (13) and (14).

We provide our numerical method with its mathematical foundation, but emphasize that it is at an early stage of development and that much remains to be done. In particular, we comment on our investigation of efficient discretization schemes for the integrals which arise in Fer streamers.

\section{Fer expansions and streamers}

We embark in this section upon the core of our argument and the essence of the novelty of its contribution, namely the elaboration of an approximation of (9)-(10) in the two uniform regimes (11) and (12). We note that it is the uniform character of our approximations which makes them a very useful tool in our endeavor to approximate small, medium or large eigenvalues of Sturm-Liouville problems.

In the following subsection, we recall Fer expansions and observe that they provide an amenable closed-form representation of the exact solution of (9)- $(10)$, with two important properties: Firstly, Fer expansions are valid whenever the potential is piecewise analytic, a feature independent of any eigenvalue. Secondly, Fer expansions are naturally defined via a recurrence relation. 
It is then, in the subsequent subsection, that we establish, under the mild conditions, (3), (4), (5) and (6), that those two properties pave the way to the uniform approximation of what we call Fer streamers: exact closed-form expressions which we devise for each of the terms appearing in Fer expansions.

\subsection{Fer expansions}

For 'small' eigenvalues, it is possible to solve $(9-(10)$ by calling upon the following definitions and theorem from (Fer, 1958), (Iserles, 1984, Theorem 3), (Iserles et al. 2000, p. 267-270).

Definition 1 Let $\mathbf{X}, \mathbf{Y} \in \mathfrak{s l}(2, \mathbb{R})$, and define the exponential, the adjoint representation, and the derivative of the adjoint representation (also referred to as the Lie bracket) as

$$
\begin{aligned}
\exp (\mathbf{X}) & :=\sum_{j=0}^{\infty} \frac{\mathbf{X}^{j}}{j !} \\
\operatorname{Ad}_{\exp (\mathbf{X})} \mathbf{Y}: & =\exp (\mathbf{X}) \mathbf{Y} \exp (-\mathbf{X}), \\
\operatorname{ad}_{\mathbf{X}} \mathbf{Y}:=:[\mathbf{X}, \mathbf{Y}]: & : \mathbf{X Y}-\mathbf{Y} \mathbf{X} .
\end{aligned}
$$

Remark 3 Note that the exponential is in $\operatorname{SL}(2, \mathbb{R})$ and that the adjoint representation and the derivative of the adjoint representation are in $\mathfrak{s l}(2, \mathbb{R})$.

Definition 2 Let $l \in \mathbb{Z}^{+}$and $t \in\left[c_{k}, c_{k+1}\right]$, and define

$$
\begin{aligned}
\mathbf{B}_{\lambda, 0}\left(c_{k}, t\right) & :=\left[\begin{array}{cr}
0 & 1 \\
q(t)-\lambda & 0
\end{array}\right], \\
\mathbf{D}_{\lambda, 0}\left(c_{k}, t\right) & :=\int_{c_{k}}^{t} \mathbf{B}_{\lambda, 0}\left(c_{k}, \xi\right) d \xi \\
\mathbf{B}_{\lambda, l}\left(c_{k}, t\right) & :=\sum_{j=1}^{\infty}(-1)^{j} \frac{j}{(j+1) !} \operatorname{ad}_{\mathbf{D}_{\lambda, l-1}\left(c_{k}, t\right)}^{j} \mathbf{B}_{\lambda, l-1}\left(c_{k}, t\right), \\
\mathbf{D}_{\lambda, l}\left(c_{k}, t\right) & :=\int_{c_{k}}^{t} \mathbf{B}_{\lambda, l}\left(c_{k}, \xi\right) d \xi .
\end{aligned}
$$

Remark 4 Observe that $\mathbf{B}_{\lambda, 0}\left(c_{k}, t\right), \mathbf{D}_{\lambda, 0}\left(c_{k}, t\right), \mathbf{B}_{\lambda, 1}\left(c_{k}, t\right), \mathbf{D}_{\lambda, 1}\left(c_{k}, t\right), \ldots \in \mathfrak{s l}(2, \mathbb{R})$. This was recognized by Zanna in (Zanna, 1996) (see the historical reference in (Iserles et al. 2000, p. 267-270)), and will go a long way to retain the geometric feature described in Remark2.

Theorem 1 ((Fer, 1958), (Iserles, 1984, Theorem 3), (Iserles et al., 2000, p. 267270)) If (3) holds true, $l \in \mathbb{Z}^{+}$and $t \in\left[c_{k}, c_{k+1}\right]$, then

$$
\begin{aligned}
& \mathbf{B}_{\lambda, 0}\left(c_{k}, t\right)=\left[\begin{array}{ll}
\mathscr{O}(1) & \mathscr{O}(1) \\
\mathscr{O}(1) & \mathscr{O}(1)
\end{array}\right], \quad t \rightarrow c_{k}^{+} \\
& \mathbf{B}_{\lambda, l}\left(c_{k}, t\right)=\left(t-c_{k}\right)^{4 \times 2^{l-1}-2}\left[\begin{array}{ll}
\mathscr{O}(1) & \mathscr{O}(1) \\
\mathscr{O}(1) & \mathscr{O}(1)
\end{array}\right], \quad t \rightarrow c_{k}^{+}
\end{aligned}
$$


and the solution of (9) is given by the Fer expansions

$$
\mathbf{Y}_{\lambda}(t)=e^{\mathbf{D}_{\lambda, 0}\left(c_{k}, t\right)} e^{\mathbf{D}_{\lambda, 1}\left(c_{k}, t\right)} e^{\mathbf{D}_{\lambda, 2}\left(c_{k}, t\right)} \cdots \mathbf{Y}_{\lambda}\left(c_{k}\right)
$$

Although Theorem 1 provides a closed-form representation of the exact solution of (9), it is not clear in practice how to evaluate or approximate (22). In particular, Theorem 1 does not provide a practical means to evaluate or approximate the infinite series (18). This state of affairs was partially resolved in (Zanna, 1998). The methodology in (Zanna, 1998) consists in two levels of truncation: one in the infinite product, and one in each infinite sum. Specifically, Zanna (1998) succeeds in approximating the exact solution by calling upon $(20)-(21)$ to discard all except the very first exponentials in the infinite product (22), and by a careful estimation of each summand to discard all except the very first terms in each infinite sum (18). This procedure works exceedingly well, for 'small' values of $|\lambda|$.

\subsection{Fer streamers}

For 'medium' or 'large' eigenvalues, however, the two-stage truncation procedure described in the previous subsection breaks down, and leads to catastrophic results. Indeed, it is possible to see that in the aforementioned procedure: $i$ ) it is only feasible to solve for eigenvalues in a compact interval $h_{\max }^{2}|\lambda| \leq 1$, as opposed to an unbounded interval, and, $i$ ) the error bounds deteriorate quite considerably, or completely, whenever $h_{\max }^{2}|\lambda| \approx 1$.

We now address this issue by proposing a different truncation of Fer expansions, which consists in one less level of truncation. Our point of departure is what we call Fer streamers: exact closed-form expressions which we devise for each infinite sum (18). With these closed-form expressions at hand, we are left only with the truncation of the infinite product (22), and we proceed by investigating the size of Fer streamers, in the two uniform regimes 11 and (12).

The result, is a numerical method which, under the mild conditions (3), (4), (5) and (6), provides a means to estimate any eigenvalue with little or no restriction on the step size! Moreover, our proposed numerical method retains the same, albeit slower, type of exponential growth in order!

\subsubsection{Closed-form expressions}

Definition 3 For every $\mathbf{X} \in \mathfrak{s l}(2, \mathbb{R})$, let

$$
\begin{aligned}
\pi(\mathbf{X}) & :=\left[\begin{array}{l}
{[\mathbf{X}]_{1,1}} \\
{[\mathbf{X}]_{1,2}} \\
{[\mathbf{X}]_{2,1}}
\end{array}\right], \\
\mathscr{C}_{\mathbf{X}} & :=\left[\begin{array}{ccc}
0 & -[\mathbf{X}]_{2,1} & {[\mathbf{X}]_{1,2}} \\
-2[\mathbf{X}]_{1,2} & 2[\mathbf{X}]_{1,1} & 0 \\
2[\mathbf{X}]_{2,1} & 0 & -2[\mathbf{X}]_{1,1}
\end{array}\right], \\
\rho(\mathbf{X}) & :=2 \sqrt{-\operatorname{det}(\mathbf{X})} .
\end{aligned}
$$


Theorem 2 If $l \in \mathbb{Z}^{+}$and $\mathbf{X}, \mathbf{Y} \in \mathfrak{s l}(2, \mathbb{R})$, then

$$
\pi\left(\operatorname{ad}_{\mathbf{X}} \mathbf{Y}\right)=\mathscr{C}_{\mathbf{X}} \pi(\mathbf{Y}), \quad \mathscr{C}_{\mathbf{X}}^{2 l-1}=\rho^{2 l-2}(\mathbf{X}) \mathscr{C}_{\mathbf{X}}, \quad \mathscr{C}_{\mathbf{X}}^{2 l}=\rho^{2 l-2}(\mathbf{X}) \mathscr{C}_{\mathbf{X}}^{2} .
$$

Proof The first assertion follows by straightforward computation, and the last two follow by induction from

$$
\mathscr{C}_{\mathbf{X}}^{3}=\rho^{2}(\mathbf{X}) \mathscr{C}_{\mathbf{X}}
$$

\section{Definition 4 Let}

$$
\psi(z):=\sum_{j=1}^{\infty}(-1)^{j} \frac{j}{(j+1) !} z^{j}=-\frac{e^{-z}\left(e^{z}-1-z\right)}{z}
$$

and

$$
\begin{gathered}
\varphi(z):=\frac{\psi(z)-\psi(-z)}{2 z}=-\sum_{j=0}^{\infty} \frac{2 j+1}{(2 j+2) !} z^{2 j}=\frac{\cosh (z)-1-z \sinh (z)}{z^{2}}, \\
\phi(z):=\frac{\psi(z)+\psi(-z)}{2 z^{2}}=\sum_{j=0}^{\infty} \frac{2 j+2}{(2 j+3) !} z^{2 j}=\frac{z \cosh (z)-\sinh (z)}{z^{3}} .
\end{gathered}
$$

Remark 5 In the sequel, it will be vital to observe that both $\varphi$ and $\phi$ are bounded along the imaginary axis:

$$
\begin{gathered}
\varphi(i x)=\sum_{j=0}^{\infty}(-1)^{j+1} \frac{2 j+1}{(2 j+2) !} x^{2 j}=\left(\frac{1-\cos (x)}{x}-\sin (x)\right) \frac{1}{x}, \\
\phi(i x)=\sum_{j=0}^{\infty}(-1)^{j} \frac{2 j+2}{(2 j+3) !} x^{2 j}=\left(\frac{\sin (x)}{x}-\cos (x)\right) \frac{1}{x^{2}} .
\end{gathered}
$$

We name the exact closed-form expressions which appear in following Theorem, as Fer streamers.

Theorem 3 If (3) holds true, $l \in \mathbb{Z}^{+}$and $t \in\left[c_{k}, c_{k+1}\right]$, then

$$
\begin{aligned}
\pi\left(\mathbf{B}_{\lambda, l}\left(c_{k}, t\right)\right)= & \varphi\left(\rho\left(\mathbf{D}_{\lambda, l-1}\left(c_{k}, t\right)\right)\right) \mathscr{C}_{\mathbf{D}_{\lambda, l-1}\left(c_{k}, t\right)} \pi\left(\mathbf{B}_{\lambda, l-1}\left(c_{k}, t\right)\right)+ \\
& +\phi\left(\rho\left(\mathbf{D}_{\lambda, l-1}\left(c_{k}, t\right)\right)\right) \mathscr{C}_{\mathbf{D}_{\lambda, l-1}\left(c_{k}, t\right)} \pi\left(\mathbf{B}_{\lambda, l-1}\left(c_{k}, t\right)\right)
\end{aligned}
$$

Proof See Appendix A 
Remark 6 As an example, since

$$
\pi\left(\mathbf{B}_{\lambda, 0}\left(c_{k}, t\right)\right)=\left[\begin{array}{c}
0 \\
1 \\
q(t)-\lambda
\end{array}\right]
$$

we have that

$$
\rho\left(\mathbf{D}_{\lambda, 0}\left(c_{k}, t\right)\right)=2\left(t-c_{k}\right) \sqrt{\frac{\int_{c_{k}}^{t} q(\xi) d \xi}{t-c_{k}}-\lambda}
$$

and that Theorem 3 yields (see Appendix B

$$
\pi\left(\mathbf{B}_{\lambda, 1}\left(c_{k}, t\right)\right)=\left[\begin{array}{c}
\varphi\left(\rho\left(\mathbf{D}_{\lambda, 0}\left(c_{k}, t\right)\right)\right) \frac{q(t)-\frac{\int_{c_{k}}^{t} q(\xi) d \xi}{t-c_{k}}}{t-c_{k}}\left(t-c_{k}\right)^{2} \\
-2 \phi\left(\rho\left(\mathbf{D}_{\lambda, 0}\left(c_{k}, t\right)\right)\right) \frac{q(t)-\frac{\int_{c_{k}}^{t} q(\xi) d \xi}{t-c_{k}}}{t-c_{k}}\left(t-c_{k}\right)^{3} \\
\frac{1}{2} \phi\left(\rho\left(\mathbf{D}_{\lambda, 0}^{t}\left(c_{k}, t\right)\right)\right) \rho^{2}\left(\mathbf{D}_{\lambda, 0}\left(c_{k}, t\right)\right) \frac{q(t)-\frac{\int_{c_{k}} q(\xi) d \xi}{t-c_{k}}}{t-c_{k}}\left(t-c_{k}\right)
\end{array}\right] .
$$

\subsubsection{Estimates}

\section{Definition 5 Let}

$$
\delta_{\left|q^{\prime}\right|}:=\max _{k \in\{0,1, \ldots, m-1\}} \max _{t \in\left(c_{k}, c_{k+1}\right)}\left\{\left|q^{\prime}(t)\right|\right\} .
$$

Theorem 4 If Assumption 1 holds true, $l \in \mathbb{Z}^{+}$and $t \in\left[c_{k}, c_{k+1}\right]$, then, in the uniform regime 11, it follows that

$$
\begin{aligned}
e^{\mathbf{D}_{\lambda, 0}\left(c_{k}, c_{k+1}\right)} \cdots e^{\mathbf{D}_{\lambda, 0}\left(a, c_{1}\right)} & =\left[\begin{array}{cc}
\mathscr{O}(1) & \mathscr{O}\left(h_{\max }\right) \\
\mathscr{O}\left(h_{\max }^{-1}\right) & \mathscr{O}(1)
\end{array}\right], \\
\pi\left(\mathbf{D}_{\lambda, l}\left(c_{k}, t\right)\right) & =\delta_{\left|q^{\prime}\right|}^{2^{l-1}} h_{\max }^{3 \times 2^{l-1}-1}\left[\begin{array}{c}
\mathscr{O}\left(h_{\max }\right) \\
\mathscr{O}\left(h_{\max }^{2}\right) \\
\mathscr{O}(1)
\end{array}\right],
\end{aligned}
$$

and, in the uniform regime (12), it follows that

$$
\begin{aligned}
e^{\mathbf{D}_{\lambda, 0}\left(c_{k}, c_{k+1}\right)} \ldots e^{\mathbf{D}_{\lambda, 0}\left(a, c_{1}\right)} & =\left[\begin{array}{cc}
\mathscr{O}(1) & \frac{\mathscr{O}(1)}{\sqrt{\lambda-q_{\max }}} \\
\mathscr{O}(1) \sqrt{\lambda-q_{\max }} & \mathscr{O}(1)
\end{array}\right] \\
\pi\left(\mathbf{D}_{\lambda, l}\left(c_{k}, t\right)\right) & =\delta_{\left|q^{\prime}\right|}^{2^{l-1} h_{\max }^{2^{l}}\left(\lambda-q_{\max }\right)^{-\frac{2^{l-1}-1}{2}}}\left[\begin{array}{c}
\frac{\mathscr{O}(1)}{\sqrt{\lambda-q_{\max }}} \\
\frac{\mathscr{O}(1)}{\lambda-q_{\max }} \\
\mathscr{O}(1)
\end{array}\right] .
\end{aligned}
$$

Proof See Appendix B 
Definition 6 Let $n \in \mathbb{Z}^{+}$, and define the

$$
\begin{aligned}
\text { exact flow: } & \mathbf{F}_{\lambda}\left(c_{k}, c_{k+1}\right) & :=\prod_{l=0}^{\infty} e^{\mathbf{D}_{\lambda, l}\left(c_{k}, c_{k+1}\right)}, \\
\text { exact solution: } & \mathbf{Y}_{\lambda}\left(c_{k+1}\right) & =\mathbf{F}_{\lambda}\left(c_{k}, c_{k+1}\right) \cdots \mathbf{F}_{\lambda}\left(c_{1}, c_{2}\right) \mathbf{F}_{\lambda}\left(a, c_{1}\right), \\
\text { approximate flow: } & \tilde{\mathbf{F}}_{\lambda, n}\left(c_{k}, c_{k+1}\right) & :=\prod_{l=0}^{n} e^{\mathbf{D}_{\lambda, l}\left(c_{k}, c_{k+1}\right)}, \\
\text { approximate solution: } & \tilde{\mathbf{Y}}_{\lambda, n}\left(c_{k+1}\right) & :=\tilde{\mathbf{F}}_{\lambda, n}\left(c_{k}, c_{k+1}\right) \cdots \tilde{\mathbf{F}}_{\lambda, n}\left(c_{1}, c_{2}\right) \tilde{\mathbf{F}}_{\lambda, n}\left(a, c_{1}\right), \\
\text { local error: } & \mathbf{L}_{\lambda, n}\left(c_{k}, c_{k+1}\right) & :=\log \left(\mathbf{F}_{\lambda}\left(c_{k}, c_{k+1}\right) \tilde{\mathbf{F}}_{\lambda, n}^{-1}\left(c_{k}, c_{k+1}\right)\right), \\
\text { global error: } & \mathbf{G}_{\lambda, n}\left(c_{k+1}\right) & :=\log \left(\mathbf{Y}_{\lambda}\left(c_{k+1}\right) \tilde{\mathbf{Y}}_{\lambda, n}^{-1}\left(c_{k+1}\right)\right) .
\end{aligned}
$$

Remark 7 Observe that Remark 4 and Definition 6 ensure that the exact flow, the exact solution, the approximate flow and the approximate solution are in $\operatorname{SL}(2, \mathbb{R})$, and that the local error and the global error are in $\mathfrak{s l}(2, \mathbb{R})$. In particular, note that the approximate solution retains the geometric feature described in Remark 2

Theorem 5 If Assumption 1 holds true, and $n \in \mathbb{Z}^{+}$, then, in the uniform regime (11), it follows that

$$
\begin{gathered}
\pi\left(\mathbf{L}_{\lambda, n}\left(c_{k}, c_{k+1}\right)\right)=\delta_{\left|q^{\prime}\right|}^{2^{n}} h_{\max }^{3 \times 2^{n}-1}\left[\begin{array}{c}
\mathscr{O}\left(h_{\max }\right) \\
\mathscr{O}\left(h_{\max }^{2}\right) \\
\mathscr{O}(1)
\end{array}\right], \\
\pi\left(\mathbf{G}_{\lambda, n}\left(c_{k+1}\right)\right)=\delta_{\left|q^{\prime}\right|}^{2^{n}} h_{\max }^{3 \times 2^{n}-2}\left[\begin{array}{c}
\mathscr{O}\left(h_{\max }\right) \\
\mathscr{O}\left(h_{\max }^{2}\right) \\
\mathscr{O}(1)
\end{array}\right],
\end{gathered}
$$

and, in the uniform regime (12), it follows that

$$
\begin{gathered}
\pi\left(\mathbf{L}_{\lambda, n}\left(c_{k}, c_{k+1}\right)\right)=\delta_{\left|q^{\prime}\right|}^{2^{n}} h_{\max }^{2^{n+1}}\left(\lambda-q_{\max }\right)^{-\frac{2^{n}-1}{2}}\left[\begin{array}{c}
\frac{\mathscr{O}(1)}{\sqrt{\lambda-q_{\max }}} \\
\frac{\mathscr{O}(1)}{\lambda-q_{\max }} \\
\mathscr{O}(1)
\end{array}\right], \\
\pi\left(\mathbf{G}_{\lambda, n}\left(c_{k+1}\right)\right)=\delta_{\left|q^{\prime}\right|}^{2^{n}} h_{\max }^{2^{n+1}-1}\left(\lambda-q_{\max }\right)^{-\frac{2^{n}-1}{2}}\left[\begin{array}{c}
\frac{\mathscr{O}(1)}{\sqrt{\lambda-q_{\max }}} \\
\frac{\mathscr{O}(1)}{\lambda-q_{\max }} \\
\mathscr{O}(1)
\end{array}\right] .
\end{gathered}
$$

Proof See Appendix C 
Corollary 1 If Assumption 1 is true, and $n \in \mathbb{Z}^{+}$, then, in the two uniform regimes (11) and (12),

$$
\begin{gathered}
\pi\left(\mathbf{L}_{\lambda, n}\left(c_{k}, c_{k+1}\right)\right)=\delta_{\left|q^{\prime}\right|}^{2^{n}} h_{\max }^{3 \times 2^{n}-1}\left[\begin{array}{c}
\mathscr{O}\left(h_{\max }\right) \\
\mathscr{O}\left(h_{\max }^{2}\right) \\
\mathscr{O}(1)
\end{array}\right], \\
\pi\left(\mathbf{G}_{\lambda, n}\left(c_{k+1}\right)\right)=\delta_{\left|q^{\prime}\right|}^{2^{n}} h_{\max }^{3 \times 2^{n}-2}\left[\begin{array}{c}
\mathscr{O}\left(h_{\max }\right) \\
\mathscr{O}\left(h_{\max }^{2}\right) \\
\mathscr{O}(1)
\end{array}\right] .
\end{gathered}
$$

\section{Numerical results}

We showcase our numerical method by computing small, medium and large eigenvalues of two Sturm-Liouville toy examples: the first is an Airy-type problem where the interval, the potential and the boundary conditions in (1) and (2) are given by

$$
a=0, \quad b=1, \quad(\forall t \in[0,1], q(t)=t), \quad \alpha_{1} \neq 0, \quad \beta_{1} \neq 0, \quad \alpha_{2}=\beta_{2}=0,
$$

i.e.,

$$
-y_{\lambda}^{\prime \prime}(t)+t y_{\lambda}(t)=\lambda y_{\lambda}(t), \quad t \in[0,1], \quad y_{\lambda}(0)=y_{\lambda}(1)=0,
$$

and the second is a Mathieu-type problem where the interval, the potential and the boundary conditions in (1) and (2) are instead given by

$a=0, \quad b=\pi, \quad(\forall t \in[0, \pi], q(t)=20 \cos (2 t)), \quad \alpha_{1} \neq 0, \quad \beta_{1} \neq 0, \quad \alpha_{2}=\beta_{2}=0$, i.e.,

$$
-y_{\lambda}^{\prime \prime}(t)+20 \cos (2 t) y_{\lambda}(t)=\lambda y_{\lambda}(t), \quad t \in[0, \pi], \quad y_{\lambda}(0)=y_{\lambda}(\pi)=0 .
$$

Since both problems have zero Dirichlet boundary conditions, it is clear that together with Remark 11, the transcendental characterization (15) yields the following nonlinear equation in the unknown eigenvalues

$$
\left\{\lambda_{j}\right\}_{j \in \mathbb{Z}_{0}^{+}}=\left\{\lambda \in\left[q_{\min },+\infty\right):\left[\mathbf{Y}_{\lambda}(b)\right]_{1,2}=0\right\} .
$$

The numerical results displayed in Figure 1 represent the absolute error between an approximation with Fer streamers and one with Matslise's (Ledoux, Daele and Berghe, 2005) package. To illustrate their power, Fer streamers were generated with the largest possible step size which satisfies Assumption 1 i.e., with

$$
m=\left\lceil(b-a) \sqrt{q_{\max }-q_{\min }}\right\rceil, \quad h_{\max }=h_{\min }=(b-a) / m,
$$

together with

$$
n=1
$$

in Corollary 1 i.e., with a global order four method. The computation of the integrals that appear in Fer streamers was done with a preliminary version of an efficient discretization scheme which will appear in a forthcoming paper (c.f., Subsection 4.1), 
and the root-finding was done with chebfun's (Driscoll, Hale and Trefethen, 2014) package. It is amazing to observe in Figure 1 that Fer streamers perform well even with extremely large step sizes: in the Airy-type problem with $h_{\max }=h_{\min }=1.00$ and in the Mathieu-type problem with $h_{\max }=h_{\min }=0.16$. On a related note, it is equally important to observe that the absolute errors in Figure 1 are decreasing with increasing $|\lambda|$, as expected in view of Theorem 5 .
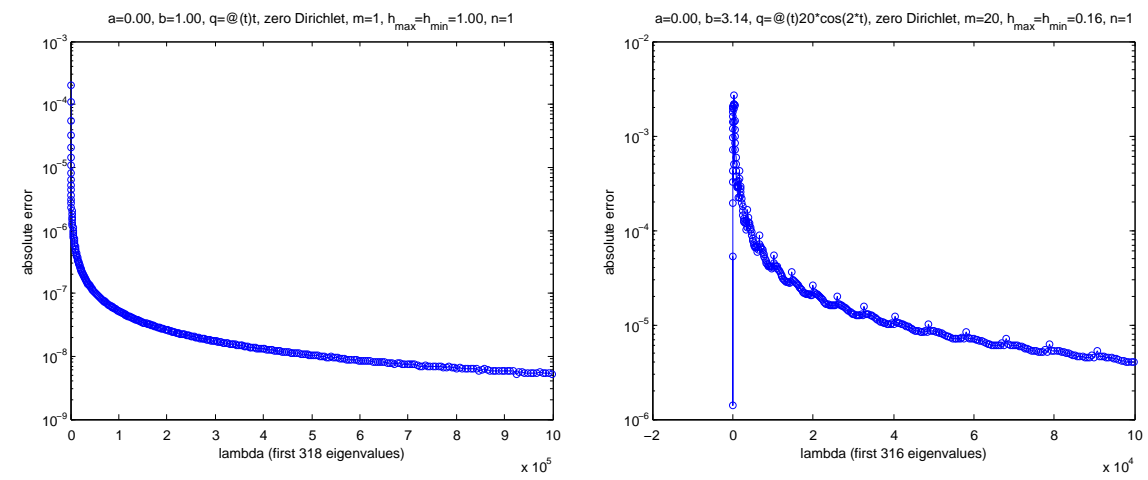

Fig. 1 Airy-type (left) and Mathieu-type (right) absolute error.

To conclude, we emphasize that together with the above analytical underpinning, it seems clear that Figure 1 depicts Fer streamers as a very promising numerical method for the computation of regular Sturm-Liouville problems in Liouville's normal form, with a continuous and piecewise analytic potential and self-adjoint separated boundary conditions.

\section{Conclusions}

We have set in motion the development of a very promising new numerical method to compute any eigenvalue of regular Sturm-Liouville problems in Liouville's normal form, with a continuous and piecewise analytic potential and self-adjoint separated boundary conditions. In particular, we have seen that the method enjoys:

- Large step sizes uniform over the whole eigenvalue range, and,

- Tight error estimates uniform for every eigenvalue.

Much remains to be done, and current and future work, which will appear in forthcoming papers, include:

- Efficient discretization schemes, and,

- Absolutely integrable potentials and self-adjoint boundary conditions. 
4.1 Efficient discretization schemes

Following Corollary 1 it is clear that $n=1,2,3, \ldots$ yields a numerical method with global order 4, 10, 22, ... uniformly w.r.t. (11) and (12). Going from theory to practice, this means that we must devise a way to compute

$$
e^{\mathbf{D}_{\lambda, 0}\left(c_{k}, c_{k+1}\right)}, e^{\mathbf{D}_{\lambda, 1}\left(c_{k}, c_{k+1}\right)}, e^{\mathbf{D}_{\lambda, 2}\left(c_{k}, c_{k+1}\right)}, \ldots, e^{\mathbf{D}_{\lambda, n}\left(c_{k}, c_{k+1}\right)},
$$

or, equivalently, given that the exponential map from $\mathfrak{s l}(2, \mathbb{R})$ to $\operatorname{SL}(2, \mathbb{R})$ has a simple closed-form expression, to compute

$$
\mathbf{D}_{\lambda, 0}\left(c_{k}, c_{k+1}\right), \mathbf{D}_{\lambda, 1}\left(c_{k}, c_{k+1}\right), \mathbf{D}_{\lambda, 2}\left(c_{k}, c_{k+1}\right), \ldots, \mathbf{D}_{\lambda, n}\left(c_{k}, c_{k+1}\right) .
$$

The first term amounts to the computation of

$$
\mathbf{D}_{\lambda, 0}\left(c_{k}, c_{k+1}\right)=\int_{c_{k}}^{c_{k+1}} \mathbf{B}_{\lambda, 0}\left(c_{k}, t\right) d t=\left[\begin{array}{cc}
0 & c_{k+1}-c_{k} \\
\int_{c_{k}}^{c_{k+1}} q(t) d t-\left(c_{k+1}-c_{k}\right) \lambda & 0
\end{array}\right],
$$

which we assume can be carried out without concern. The computation of the other terms is sketched below.

\subsubsection{A method with global order 4}

Calling upon Corollary 1 , it is clear that $n=1$ results in a numerical method with local order 5 and global order 4 uniformly w.r.t. (11) and (12). It is now important to emphasize the intricacies associated with the computation of

$$
\mathbf{D}_{\lambda, 1}\left(c_{k}, c_{k+1}\right)=\int_{c_{k}}^{c_{k+1}} \mathbf{B}_{\lambda, 1}\left(c_{k}, t\right) d t .
$$

To this end, we begin by partitioning (4) and (5) into three intervals:

$$
\lambda \in\left[q_{\max }-h_{\max }^{-2}, q_{\min }-1\right] \cup\left[q_{\min }-1, q_{\max }+1\right] \cup\left[q_{\max }+1,+\infty\right) .
$$

Focusing first on the right interval in 23], we note that a closer investigation reveals that (c.f., Remark6 and Definition 4 )

$$
\begin{aligned}
\mathbf{B}_{\lambda, 1}\left(c_{k}, t\right)= & \eta_{\lambda, 1}^{\prime}\left(c_{k}, t\right) \mathbf{R}_{1}+ \\
& +\mathbf{S}_{\lambda, 1}\left(c_{k}, t\right) e^{i 2 \sqrt{\lambda-q\left(c_{k}^{+}\right)}\left(t-c_{k}\right)}+\overline{\mathbf{S}_{\lambda, 1}\left(c_{k}, t\right)} e^{-i 2 \sqrt{\lambda-q\left(c_{k}^{+}\right)}\left(t-c_{k}\right)},
\end{aligned}
$$

where the derivatives $\eta_{\lambda, 1}^{(j)}\left(c_{k}, t\right) \in \mathbb{R}$ and $\mathbf{S}_{\lambda, 1}^{(j)}\left(c_{k}, t\right) \in \mathfrak{s l}(2, \mathbb{C})$ can be bounded independently of $\lambda$ and $\mathbf{R}_{1} \in \mathfrak{s l}(2, \mathbb{R})$, which, in turn, implies that

$$
\begin{aligned}
\mathbf{D}_{\lambda, 1}\left(c_{k}, c_{k+1}\right)= & \left(\eta_{\lambda, 1}\left(c_{k}, c_{k+1}\right)-\eta_{\lambda, 1}\left(c_{k}, c_{k}\right)\right) \mathbf{R}_{1}+ \\
& +2 \mathfrak{R}\left(\int_{c_{k}}^{c_{k+1}} \mathbf{S}_{\lambda, 1}\left(c_{k}, t\right) e^{i 2 \sqrt{\lambda-q\left(c_{k}^{+}\right)}\left(t-c_{k}\right)} d t\right)
\end{aligned}
$$

amounts to the computation of a one-dimensional mildly to highly oscillatory Fouriertype integral. This observation is key since it is well-known that standard techniques 
such as Gauss-Christoffel quadrature are useless in the presence of mildly to highly oscillatory behaviour, and specialized techniques must be used instead. Here we choose a Filon-type quadrature (consistent with the local order of the numerical method), because it performs well not only in the highly oscillatory regime, but also in the mildly oscillatory regime. Looking next at the middle interval in (23), we write (c.f., Remark6 and Definition 4)

$$
\begin{aligned}
& \mathbf{B}_{\lambda, 1}\left(c_{k}, t\right)= \\
& =\left(q(t)-\frac{\int_{c_{k}}^{t} q(\xi) d \xi}{t-c_{k}}\right) \times \\
& \quad \times\left[\begin{array}{cc}
\left.\varphi\left(\sqrt{\rho^{2}\left(\mathbf{D}_{\lambda, 0}\left(c_{k}, t\right)\right.}\right)\right)\left(t-c_{k}\right) & -2 \phi\left(\sqrt{\rho^{2}\left(\mathbf{D}_{\lambda, 0}\left(c_{k}, t\right)\right)}\right)\left(t-c_{k}\right)^{2} \\
\frac{1}{2} \phi\left(\sqrt{\rho^{2}\left(\mathbf{D}_{\lambda, 0}\left(c_{k}, t\right)\right)}\right) \rho^{2}\left(\mathbf{D}_{\lambda, 0}\left(c_{k}, t\right)\right) & -\varphi\left(\sqrt{\rho^{2}\left(\mathbf{D}_{\lambda, 0}\left(c_{k}, t\right)\right)}\right)\left(t-c_{k}\right)
\end{array}\right],
\end{aligned}
$$

where $\varphi(\sqrt{z})$ and $\phi(\sqrt{z})$ are analytic in $z \in \mathbb{C}$, and,

$$
\rho^{2}\left(\mathbf{D}_{\lambda, 0}\left(c_{k}, t\right)\right)=4\left(t-c_{k}\right)^{2}\left(\frac{\int_{c_{k}}^{t} q(\xi) d \xi}{t-c_{k}}-\lambda\right)
$$

and note that, since $\lambda$ is confined to a fixed compact interval,

$$
\mathbf{D}_{\lambda, 1}\left(c_{k}, c_{k+1}\right)=\int_{c_{k}}^{c_{k+1}} \mathbf{B}_{\lambda, 1}\left(c_{k}, t\right) d t
$$

amounts to the computation of a one-dimensional well-behaved integral, which can be uniformly discretized with either a Gauss-Legendre or a Filon-type quadrature (consistent with the local order of the numerical method). Looking next at the left interval in 23, we write (c.f., Remark6 and Definition 4)

$$
\begin{aligned}
\mathbf{B}_{\lambda, 1}\left(c_{k}, t\right)= & \eta_{\lambda, 1}^{\prime}\left(c_{k}, t\right) \mathbf{R}_{1}+ \\
& +\mathbf{U}_{\lambda, 1}\left(c_{k}, t\right) e^{2 \sqrt{q\left(c_{k}^{+}\right)-\lambda}\left(t-c_{k}\right)}+\mathbf{V}_{\lambda, 1}\left(c_{k}, t\right) e^{-2 \sqrt{q\left(c_{k}^{+}\right)-\lambda\left(t-c_{k}\right)},}
\end{aligned}
$$

where the derivatives $\eta_{\lambda, 1}^{(j)}\left(c_{k}, t\right) \in \mathbb{R}, \mathbf{U}_{\lambda, 1}^{(j)}\left(c_{k}, t\right) \in \mathfrak{s l}(2, \mathbb{R})$ and $\mathbf{V}_{\lambda, 1}^{(j)}\left(c_{k}, t\right) \in \mathfrak{s l}(2, \mathbb{R})$ can be bounded independently of $\lambda$ and $\mathbf{R}_{1} \in \mathfrak{s l}(2, \mathbb{R})$, and note that

$$
\begin{aligned}
& \mathbf{D}_{\lambda, 1}\left(c_{k}, c_{k+1}\right)= \\
& =\left(\eta_{\lambda, 1}\left(c_{k}, c_{k+1}\right)-\eta_{\lambda, 1}\left(c_{k}, c_{k}\right)\right) \mathbf{R}_{1}+ \\
& \quad+\int_{c_{k}}^{c_{k+1}} \mathbf{U}_{\lambda, 1}\left(c_{k}, t\right) e^{2 \sqrt{q\left(c_{k}^{+}\right)-\lambda\left(t-c_{k}\right)}} d t+\int_{c_{k}}^{c_{k+1}} \mathbf{V}_{\lambda, 1}\left(c_{k}, t\right) e^{-2 \sqrt{q\left(c_{k}^{+}\right)-\lambda\left(t-c_{k}\right)}} d t
\end{aligned}
$$

amounts to the computation of one-dimensional exponentially increasing and decreasing integrals with linear exponent. This observation is key since it is wellknown that standard techniques such as Gauss-Christoffel quadrature are useless in the presence of exponentially increasing and decreasing behaviour, and specialized techniques must be used instead. Here we choose a Filon-type quadrature (consistent with the local order of the numerical method), because it performs well regardless of the sign and magnitude of the exponent. 


\subsubsection{A method with global order 10}

Calling upon Corollary 1 it is clear that $n=2$ results in a numerical method with local order 11 and global order 10 uniformly w.r.t. (11) and (12). It is now important to draw attention to the intricacies associated with the computation of

$$
\mathbf{D}_{\lambda, 2}\left(c_{k}, c_{k+1}\right)=\int_{c_{k}}^{c_{k+1}} \mathbf{B}_{\lambda, 2}\left(c_{k}, t\right) d t .
$$

To this end, we begin by estimating in the two uniform regimes (11) and (12):

$$
\begin{aligned}
& \mathbf{D}_{\lambda, 2}\left(c_{k}, c_{k+1}\right)= \\
& =\int_{c_{k}}^{c_{k+1}} \mathbf{B}_{\lambda, 2}\left(c_{k}, t\right) d t \\
& =\int_{c_{k}}^{c_{k+1}} \varphi\left(\rho\left(\mathbf{D}_{\lambda, 1}\left(c_{k}, t\right)\right)\right) \operatorname{ad}_{\mathbf{D}_{\lambda, 1}\left(c_{k}, t\right)} \mathbf{B}_{\lambda, 1}\left(c_{k}, t\right) d t+ \\
& +\int_{c_{k}}^{c_{k+1}} \phi\left(\rho\left(\mathbf{D}_{\lambda, 1}\left(c_{k}, t\right)\right)\right) \operatorname{ad}_{\mathbf{D}_{\lambda, 1}\left(c_{k}, t\right)}^{2} \mathbf{B}_{\lambda, 1}\left(c_{k}, t\right) d t \\
& =-\frac{1}{2} \int_{c_{k}}^{c_{k+1}} \int_{c_{k}}^{t_{1}}\left[\mathbf{B}_{\lambda, 1}\left(c_{k}, t_{2}\right), \mathbf{B}_{\lambda, 1}\left(c_{k}, t_{1}\right)\right] d t_{2} d t_{1}+ \\
& +\frac{1}{3} \int_{c_{k}}^{c_{k+1}} \int_{c_{k}}^{t_{1}} \int_{c_{k}}^{t_{1}}\left[\mathbf{B}_{\lambda, 1}\left(c_{k}, t_{3}\right),\left[\mathbf{B}_{\lambda, 1}\left(c_{k}, t_{2}\right), \mathbf{B}_{\lambda, 1}\left(c_{k}, t_{1}\right)\right]\right] d t_{3} d t_{2} d t_{1}+ \\
& +\delta_{\left|q^{\prime}\right|}^{4} h_{\max }^{11}\left[\begin{array}{cc}
\mathscr{O}\left(h_{\max }\right) & \mathscr{O}\left(h_{\max }^{2}\right) \\
\mathscr{O}(1) & -\mathscr{O}\left(h_{\max }\right)
\end{array}\right] \text {, }
\end{aligned}
$$

where the second equality is due to Theorem 3 and the third equality is due to Definition 4 and Theorem 4 Focusing first on the right interval in 23 , we note that inserting (24) into the previous estimate, and collecting like terms, leads to the computation of two-dimensional and three-dimensional mildly to highly oscillatory Fourier-type integrals, which can be uniformly discretized with a Filon-type quadrature (consistent with the local order of the numerical method). Looking next at the middle interval in (23), we note that inserting (25) into the previous estimate leads to the computation of two-dimensional and three-dimensional well-behaved integrals, which can be uniformly discretized with either a Gauss-Legendre or a Filon-type quadrature (consistent with the local order of the numerical method). Looking next at the left interval in (23), we note that inserting (26) into the previous estimate, and collecting like terms, leads to the computation of two-dimensional and three-dimensional exponentially increasing and decreasing integrals with linear exponent, which can be uniformly discretized with a Filon-type quadrature (consistent with the local order of the numerical method).

\subsubsection{Methods with global order greater than or equal to 22}

Calling upon Corollary 1 , it is clear that $n=3,4,5, \ldots$ results in a numerical method with global order $22,46,94, \ldots$ uniformly w.r.t. (11) and (12), and the question arises of whether it is also possible to develop efficient discretization schemes for these cases; a matter currently being investigated. 


\subsubsection{The challenges}

The challenges include:

- Tracking down the behaviour of each multivariate integrand, by collecting like terms, in order to apply each Filon-type quadrature successfully,

- Choosing the number of quadrature points of each Filon-type quadrature conscientiously in order to be consistent with the local order of the numerical method,

- Choosing the set of quadrature points of each Filon-type quadratures intelligently in order to minimize the local and global error estimates whenever possible,

- Decreasing the number of function evaluations as a means to reduce the computational effort,

- Decreasing the amount of linear algebra as a means to reduce the computational effort, and,

- Comparing well-developed efficient discretization schemes for Sturm-Liouville problems based on Fer streamers with other well-developed software for SturmLiouville problems based on different techniques.

\subsection{Absolutely integrable potentials and self-adjoint boundary conditions}

Having developed Fer streamers and started the investigation of efficient discretization schemes for their implementation, we are now also looking forward to extending Fer streamers to regular Sturm-Liouville problems in Liouville's normal form, with absolutely integrable potentials and self-adjoint separated, real coupled or complex coupled boundary conditions.

\section{A Proof of Theorem 3}

Note that

$$
\begin{aligned}
\pi & \left(\mathbf{B}_{\lambda, l}\left(c_{k}, t\right)\right)= \\
= & \pi\left(\sum_{j=1}^{\infty}(-1)^{j} \frac{j}{(j+1) !} \mathrm{ad}_{\mathbf{D}_{\lambda, l-1}^{j}\left(c_{k}, t\right)}^{j} \mathbf{B}_{\lambda, l-1}\left(c_{k}, t\right)\right) \\
= & \sum_{j=1}^{\infty}(-1)^{j} \frac{j}{(j+1) !} \pi\left(\operatorname{ad}_{\mathbf{D}_{\lambda, l-1}^{j}\left(c_{k}, t\right)}^{j} \mathbf{B}_{\lambda, l-1}\left(c_{k}, t\right)\right) \\
= & \left(\sum_{j=1}^{\infty}(-1)^{j} \frac{j}{(j+1) !} \mathscr{C}_{\mathbf{D}_{\lambda, l-1}^{j}\left(c_{k}, t\right)}^{j}\right) \pi\left(\mathbf{B}_{\lambda, l-1}\left(c_{k}, t\right)\right) \\
= & -\left(\sum_{j=1}^{\infty} \frac{2 j-1}{(2 j) !} \mathscr{C}_{\mathbf{D}_{\lambda, l-1}\left(c_{k}, t\right)}^{2 j-1}\right) \pi\left(\mathbf{B}_{\lambda, l-1}\left(c_{k}, t\right)\right)+ \\
& +\left(\sum_{j=1}^{\infty} \frac{2 j}{(2 j+1) !} \mathscr{C}_{\mathbf{D}_{\lambda, l-1}^{2 j}\left(c_{k}, t\right)}^{2 j}\right) \pi\left(\mathbf{B}_{\lambda, l-1}\left(c_{k}, t\right)\right)
\end{aligned}
$$




$$
\begin{aligned}
= & -\left(\sum_{j=1}^{\infty} \frac{2 j-1}{(2 j) !} \rho^{2 j-2}\left(\mathbf{D}_{\lambda, l-1}\left(c_{k}, t\right)\right)\right) \mathscr{C}_{\mathbf{D}_{\lambda, l-1}\left(c_{k}, t\right)} \pi\left(\mathbf{B}_{\lambda, l-1}\left(c_{k}, t\right)\right)+ \\
& +\left(\sum_{j=1}^{\infty} \frac{2 j}{(2 j+1) !} \rho^{2 j-2}\left(\mathbf{D}_{\lambda, l-1}\left(c_{k}, t\right)\right)\right) \mathscr{C}_{\mathbf{D}_{\lambda, l-1}\left(c_{k}, t\right)}^{2} \pi\left(\mathbf{B}_{\lambda, l-1}\left(c_{k}, t\right)\right)
\end{aligned}
$$

where the first equality is due to Definition 2, and the third and last equalities are due to Theorem 2 .

\section{B Proof of Theorem 4}

Recall Definitions 2 and 3 and note that

$$
\rho\left(\mathbf{D}_{\lambda, 0}\left(c_{k}, t\right)\right)=2\left(t-c_{k}\right) \sqrt{\frac{\int_{c_{k}}^{t} q(\xi) d \xi}{t-c_{k}}-\lambda} .
$$

Note further that, (27) and assumptions (4) and (5) ensure

$$
\begin{aligned}
\lambda \in\left[q_{\max }-h_{\max }^{-2}, q_{\min }\right] & \Rightarrow \rho\left(\mathbf{D}_{\lambda, 0}\left(c_{k}, t\right)\right) \in\left[0,2 h_{\max } \sqrt{q_{\max }-\lambda}\right] \subseteq[0,2] \\
\lambda \in\left[q_{\min }, q_{\max }\right] & \Rightarrow\left|\rho\left(\mathbf{D}_{\lambda, 0}\left(c_{k}, t\right)\right)\right| \leq 2 h_{\max } \sqrt{q_{\max }-q_{\min }} \leq 2 \\
\lambda \in\left[q_{\max }, q_{\max }+h_{\max }^{-2}\right] & \Rightarrow \rho\left(\mathbf{D}_{\lambda, 0}\left(c_{k}, t\right)\right) \in i\left[0,2 h_{\max } \sqrt{\lambda-q_{\min }}\right] \subseteq i[0,2 \sqrt{2}] \\
\lambda \in\left[q_{\max }+h_{\max }^{-2},+\infty\right) & \Rightarrow \rho\left(\mathbf{D}_{\lambda, 0}\left(c_{k}, t\right)\right) \in i\left[2\left(t-c_{k}\right) \sqrt{\lambda-q_{\max }},+\infty\right)
\end{aligned}
$$

which, together with Definition 4 and Remark 5, lead to the following estimates, in the two uniform regimes (11) and (12):

$$
\begin{aligned}
\left|\varphi\left(\rho\left(\mathbf{D}_{\lambda, 0}\left(c_{k}, t\right)\right)\right)\right| & \leq 2 \text {, w.r.t } 11], \\
\left|\phi\left(\rho\left(\mathbf{D}_{\lambda, 0}\left(c_{k}, t\right)\right)\right)\right| & \leq 1 \text {, w.r.t } 11], \\
\left|\phi\left(\rho\left(\mathbf{D}_{\lambda, 0}\left(c_{k}, t\right)\right)\right) \rho^{2}\left(\mathbf{D}_{\lambda, 0}\left(c_{k}, t\right)\right)\right| & \leq 2 \text {, w.r.t }[11, \\
\left|\varphi\left(\rho\left(\mathbf{D}_{\lambda, 0}\left(c_{k}, t\right)\right)\right)\right| & \leq \frac{\left(t-c_{k}\right)^{-1}}{\sqrt{\lambda-q_{\max }}}, \text { w.r.t [12), } \\
\left|\phi\left(\rho\left(\mathbf{D}_{\lambda, 0}\left(c_{k}, t\right)\right)\right)\right| & \leq \frac{1}{2} \frac{\left(t-c_{k}\right)^{-2}}{\lambda-q_{\max }}, \text { w.r.t }[12), \\
\left|\phi\left(\rho\left(\mathbf{D}_{\lambda, 0}\left(c_{k}, t\right)\right)\right) \rho^{2}\left(\mathbf{D}_{\lambda, 0}\left(c_{k}, t\right)\right)\right| & \leq 2, \text { w.r.t }[12) .
\end{aligned}
$$


B.1 Estimating $\exp \left(\mathbf{D}_{\lambda, 0}\left(c_{k}, c_{k+1}\right)\right) \cdots \exp \left(\mathbf{D}_{\lambda, 0}\left(a, c_{1}\right)\right)$

Firstly, in the uniform regime (11), we have

$$
\begin{aligned}
& e^{\mathbf{D}_{\lambda, 0}\left(c_{k}, c_{k+1}\right)}= \\
& =\cosh \frac{\rho\left(\mathbf{D}_{\lambda, 0}\left(c_{k}, c_{k+1}\right)\right)}{2}\left[\begin{array}{ll}
1 & 0 \\
0 & 1
\end{array}\right]+
\end{aligned}
$$

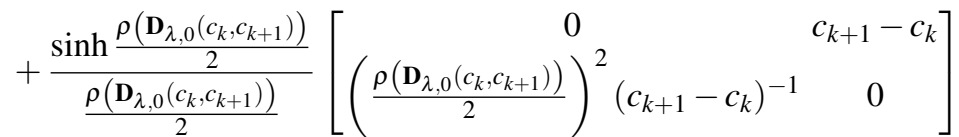

$$
\begin{aligned}
& =\mathscr{O}(1)\left[\begin{array}{ll}
1 & 0 \\
0 & 1
\end{array}\right]+\mathscr{O}(1)\left[\begin{array}{cc}
0 & \mathscr{O}(1) h_{\max } \\
\mathscr{O}(1) h_{\min }^{-1} & 0
\end{array}\right] \\
& =\mathscr{O}(1)\left[\begin{array}{ll}
1 & 0 \\
0 & 1
\end{array}\right]+\mathscr{O}(1)\left[\begin{array}{cc}
0 & \mathscr{O}(1) h_{\max } \\
\mathscr{O}(1) h_{\max }^{-1} & 0
\end{array}\right]
\end{aligned}
$$

where we have called upon (6), 28), 29) and (30). Secondly, in the uniform regime (12), we have

$$
\begin{aligned}
& e^{\mathbf{D}_{\lambda, 0}\left(c_{k}, c_{k+1}\right)}= \\
& =\cos \frac{\rho\left(\mathbf{D}_{\lambda, 0}\left(c_{k}, c_{k+1}\right)\right)}{2 i}\left[\begin{array}{ll}
1 & 0 \\
0 & 1
\end{array}\right]+ \\
& +\sin \frac{\rho\left(\mathbf{D}_{\lambda, 0}\left(c_{k}, c_{k+1}\right)\right)}{2 i}\left[\begin{array}{cc}
0 & \frac{c_{k+1}-c_{k}}{(2 i)^{-1} \rho\left(\mathbf{D}_{\lambda, 0}\left(c_{k}, c_{k+1}\right)\right)} \\
-\frac{(2 i)^{-1} \rho\left(\mathbf{D}_{\lambda, 0}\left(c_{k}, c_{k+1}\right)\right)}{c_{k+1}-c_{k}} & 0
\end{array}\right] \\
& =\mathscr{O}(1)\left[\begin{array}{ll}
1 & 0 \\
0 & 1
\end{array}\right]+\mathscr{O}(1)\left[\begin{array}{cc}
0 & \frac{1}{\sqrt{\lambda-\frac{\left.\int_{c_{k}+1}^{c_{k}} q\right) d \xi}{c_{k+1}-c_{k}}}} \\
-\sqrt{\lambda-\frac{\int_{c_{k}}^{c_{k+1}} q(\xi) d \xi}{c_{k+1}-c_{k}}} & 0
\end{array}\right] \\
& =\mathscr{O}(1)\left[\begin{array}{ll}
1 & 0 \\
0 & 1
\end{array}\right]+\mathscr{O}(1)\left[\begin{array}{cc}
0 & \mathscr{O}(1) \frac{1}{\sqrt{\lambda-q_{\max }}} \\
\mathscr{O}(1) \sqrt{\lambda-q_{\min }} & 0
\end{array}\right] \\
& =\mathscr{O}(1)\left[\begin{array}{ll}
1 & 0 \\
0 & 1
\end{array}\right]+\mathscr{O}(1)\left[\begin{array}{cc}
0 & \mathscr{O}(1) \frac{1}{\sqrt{\lambda-q_{\max }}} \\
\mathscr{O}(1) \sqrt{\lambda-q_{\max }} & 0
\end{array}\right]
\end{aligned}
$$

where the second equality is due to [27) and (31), and the last equality is due to the fact that (4) ensures that

$$
\frac{\sqrt{\lambda-q_{\min }}}{\sqrt{\lambda-q_{\max }}}=\sqrt{1+\frac{q_{\max }-q_{\min }}{\lambda-q_{\max }}} \leq \sqrt{1+h_{\max }^{2}\left(q_{\max }-q_{\min }\right)} \leq \sqrt{2}
$$


B.2 Estimating $\pi\left(\mathbf{B}_{\lambda, 1}\left(c_{k}, t\right)\right)$ and $\pi\left(\mathbf{D}_{\lambda, 1}\left(c_{k}, t\right)\right)$

Finally, we note that $(32)-(37)$, in turn, imply that

$$
\begin{aligned}
& \varphi\left(\rho\left(\mathbf{D}_{\lambda, 0}\left(c_{k}, t\right)\right)\right) \mathscr{C}_{\mathbf{D}_{\lambda, 0}\left(c_{k}, t\right)} \pi\left(\mathbf{B}_{\lambda, 0}\left(c_{k}, t\right)\right)= \\
& =\left[\begin{array}{c}
\varphi\left(\rho\left(\mathbf{D}_{\lambda, 0}\left(c_{k}, t\right)\right)\right) \frac{q(t)-\frac{\int_{c_{k}}^{t} q(\xi) d \xi}{t-c_{k}}}{t-c_{k}}\left(t-c_{k}\right)^{2} \\
0 \\
0
\end{array}\right] \\
& =\left\{\begin{array}{c}
\delta_{\left|q^{\prime}\right|}\left[\begin{array}{c}
\mathscr{O}\left(h_{\text {max }}^{2}\right) \\
0 \\
0
\end{array}\right], \text { w.r.t }[11), \\
\delta_{\left|q^{\prime}\right|}\left[\begin{array}{c}
\mathscr{O}\left(h_{\max }\right)\left(\lambda-q_{\max }\right)^{-\frac{1}{2}} \\
0 \\
0
\end{array}\right], \text { w.r.t }[12),
\end{array}\right.
\end{aligned}
$$

and

$$
\begin{aligned}
& \phi\left(\rho\left(\mathbf{D}_{\lambda, 0}\left(c_{k}, t\right)\right)\right) \mathscr{C}_{\mathbf{D}_{\lambda, 0}\left(c_{k}, t\right)}^{2} \pi\left(\mathbf{B}_{\lambda, 0}\left(c_{k}, t\right)\right)= \\
& 0 \\
& =\left[\begin{array}{c}
0 \\
-2 \phi\left(\rho\left(\mathbf{D}_{\lambda, 0}\left(c_{k}, t\right)\right)\right) \frac{q(t)-\frac{\int_{c_{k}}^{t} q(\xi) d \xi}{t-c_{k}}}{t-c_{k}}\left(t-c_{k}\right)^{3} \\
\frac{1}{2} \phi\left(\rho\left(\mathbf{D}_{\lambda, 0}\left(c_{k}, t\right)\right)\right) \rho^{2}\left(\mathbf{D}_{\lambda, 0}\left(c_{k}, t\right)\right) \frac{q(t)-\frac{\int_{c_{k}} q(\xi) d \xi}{t-c_{k}}}{t-c_{k}}\left(t-c_{k}\right)
\end{array}\right] \\
& =\left\{\begin{array}{c}
0 \\
\delta_{\left|q^{\prime}\right|}\left[\begin{array}{c}
0 \\
\mathscr{O}\left(h_{\max }^{3}\right) \\
\mathscr{O}\left(h_{\max }\right)
\end{array}\right], \text { w.r.t }[11), \\
0 \\
\delta_{\left|q^{\prime}\right|}\left[\begin{array}{c}
\mathscr{O}\left(h_{\max }\right)\left(\lambda-q_{\max }\right)^{-1} \\
\mathscr{O}\left(h_{\max }\right)
\end{array}\right], \text { w.r.t }[12),
\end{array}\right.
\end{aligned}
$$

which, according to Theorem 3 , lead to

$$
\begin{aligned}
& \pi\left(\mathbf{B}_{\lambda, 1}\left(c_{k}, t\right)\right)=\varphi\left(\rho\left(\mathbf{D}_{\lambda, 0}\left(c_{k}, t\right)\right)\right) \mathscr{C}_{\mathbf{D}_{\lambda, 0}\left(c_{k}, t\right)} \pi\left(\mathbf{B}_{\lambda, 0}\left(c_{k}, t\right)\right)+ \\
& +\phi\left(\rho\left(\mathbf{D}_{\lambda, 0}\left(c_{k}, t\right)\right)\right) \mathscr{C}_{\mathbf{D}_{\lambda, 0}\left(c_{k}, t\right)}^{2} \pi\left(\mathbf{B}_{\lambda, 0}\left(c_{k}, t\right)\right) \\
& =\left\{\begin{array}{c}
\delta_{\left|q^{\prime}\right|}\left[\begin{array}{c}
\mathscr{O}\left(h_{\max }^{2}\right) \\
\mathscr{O}\left(h_{\max }^{3}\right) \\
\mathscr{O}\left(h_{\max }\right)
\end{array}\right], \text { w.r.t }[11, \\
\delta_{\left|q^{\prime}\right|}\left[\begin{array}{c}
\mathscr{O}\left(h_{\max }\right)\left(\lambda-q_{\max }\right)^{-\frac{1}{2}} \\
\mathscr{O}\left(h_{\max }\right)\left(\lambda-q_{\max }\right)^{-1} \\
\mathscr{O}\left(h_{\max }\right)
\end{array}\right], \text { w.r.t }[12, .
\end{array}\right.
\end{aligned}
$$


B.3 Estimating $\pi\left(\mathbf{B}_{\lambda, l}\left(c_{k}, t\right)\right)$ and $\pi\left(\mathbf{D}_{\lambda, l}\left(c_{k}, t\right)\right)$ for $l \geq 2$

Follows by induction.

\section{B.3.1 First step: $l=2$}

Given Definition 4 and the uniform estimates for $\pi\left(\mathbf{B}_{\lambda, 1}\left(c_{k}, t\right)\right)$ in the previous subsection, it is now clear that

$$
\begin{gathered}
\varphi\left(\rho\left(\mathbf{D}_{\lambda, 1}\left(c_{k}, t\right)\right)\right)=\left\{\begin{array}{l}
-\frac{1}{2}+\delta_{\left|q^{\prime}\right|}^{2} \mathscr{O}\left(h_{\max }^{6}\right), \text { w.r.t }[11), \\
-\frac{1}{2}+\delta_{\left|q^{\prime}\right|}^{2} \mathscr{O}\left(h_{\max }^{4}\right)\left(\lambda-q_{\max }\right)^{-1}, \text { w.r.t }[12),
\end{array}\right. \\
\phi\left(\rho\left(\mathbf{D}_{\lambda, 1}\left(c_{k}, t\right)\right)\right)=\left\{\begin{array}{l}
\frac{1}{3}+\delta_{\left|q^{\prime}\right|}^{2} \mathscr{O}\left(h_{\max }^{6}\right), \text { w.r.t }[11), \\
\frac{1}{3}+\delta_{\left|q^{\prime}\right|}^{2} \mathscr{O}\left(h_{\max }^{4}\right)\left(\lambda-q_{\max }\right)^{-1}, \text { w.r.t }[12,
\end{array}\right.
\end{gathered}
$$

and, according to Theorem 3 , that

$$
\begin{aligned}
\pi\left(\mathbf{B}_{\lambda, 2}\left(c_{k}, t\right)\right)= & \varphi\left(\rho\left(\mathbf{D}_{\lambda, 1}\left(c_{k}, t\right)\right)\right) \mathscr{C}_{\mathbf{D}_{\lambda, 1}\left(c_{k}, t\right)} \pi\left(\mathbf{B}_{\lambda, 1}\left(c_{k}, t\right)\right)+ \\
& +\phi\left(\rho\left(\mathbf{D}_{\lambda, 1}\left(c_{k}, t\right)\right)\right) \mathscr{C}_{\mathbf{D}_{\lambda, 1}\left(c_{k}, t\right)}^{2} \pi\left(\mathbf{B}_{\lambda, 1}\left(c_{k}, t\right)\right) \\
= & \left\{\begin{array}{l}
\delta_{\left|q^{\prime}\right|}^{2}\left[\begin{array}{l}
\mathscr{O}\left(h_{\max }^{5}\right) \\
\mathscr{O}\left(h_{\max }^{6}\right) \\
\mathscr{O}\left(h_{\max }^{4}\right)
\end{array}\right], \text { w.r.t }[11, \\
\delta_{\left|q^{\prime}\right|}^{2}\left[\begin{array}{l}
\mathscr{O}\left(h_{\max }^{3}\right)\left(\lambda-q_{\max }\right)^{-1} \\
\mathscr{O}\left(h_{\max }^{3}\right)\left(\lambda-q_{\max }\right)^{-\frac{3}{2}} \\
\mathscr{O}\left(h_{\max }^{3}\right)\left(\lambda-q_{\max }\right)^{-\frac{1}{2}}
\end{array}\right], \text { w.r.t }[12) .
\end{array}\right.
\end{aligned}
$$

B.3.2 Induction step: $l \Rightarrow l+1$

Given the induction claim, it is now clear that

$$
\begin{gathered}
\varphi\left(\rho\left(\mathbf{D}_{\lambda, l}\left(c_{k}, t\right)\right)\right)=\left\{\begin{array}{l}
-\frac{1}{2}+\delta_{\left|q^{\prime}\right|}^{2^{l}} \mathscr{O}\left(h_{\max }^{3 \times 2^{l}}\right), \text { w.r.t }[11, \\
-\frac{1}{2}+\delta_{\left|q^{\prime}\right|}^{2^{l}} \mathscr{O}\left(h_{\max }^{2^{l+1}}\right)\left(\lambda-q_{\max }\right)^{-2^{l-1}}, \text { w.r.t }[12),
\end{array}\right. \\
\phi\left(\rho\left(\mathbf{D}_{\lambda, l}\left(c_{k}, t\right)\right)\right)=\left\{\begin{array}{l}
\frac{1}{3}+\delta_{\left|q^{\prime}\right|}^{2^{l}} \mathscr{O}\left(h_{\max }^{3 \times 2^{l}}\right), \text { w.r.t }[11, \\
\frac{1}{3}+\delta_{\left|q^{\prime}\right|}^{2^{l}} \mathscr{O}\left(h_{\max }^{2^{l+1}}\right)\left(\lambda-q_{\max }\right)^{-2^{l-1}}, \text { w.r.t }[12,
\end{array}\right.
\end{gathered}
$$


and, according to Theorem 3 , that

$$
\begin{aligned}
& \pi\left(\mathbf{B}_{\lambda, l+1}\left(c_{k}, t\right)\right)=\varphi\left(\rho\left(\mathbf{D}_{\lambda, l}\left(c_{k}, t\right)\right)\right) \mathscr{C}_{\mathbf{D}_{\lambda, l}\left(c_{k}, t\right)} \pi\left(\mathbf{B}_{\lambda, l}\left(c_{k}, t\right)\right)+ \\
& +\phi\left(\rho\left(\mathbf{D}_{\lambda, l}\left(c_{k}, t\right)\right)\right) \mathscr{C}_{\mathbf{D}_{\lambda, l}\left(c_{k}, t\right)}^{2} \pi\left(\mathbf{B}_{\lambda, l}\left(c_{k}, t\right)\right) \\
& =\left\{\begin{aligned}
\delta_{\left|q^{\prime}\right|}^{2^{l}}\left[\begin{array}{c}
\mathscr{O}\left(h_{\max }^{3 \times 2^{l}-1}\right) \\
\mathscr{O}\left(h_{\max }^{3 \times 2^{l}}\right) \\
\mathscr{O}\left(h_{\max }^{3 \times 2}-2\right)
\end{array}\right], \text { w.r.t [11), } \\
\delta_{\left|q^{\prime}\right|}^{2^{l}}\left[\begin{array}{c}
\mathscr{O}\left(h_{\max }^{2^{l+1}-1}\right)\left(\lambda-q_{\max }\right)^{-\frac{2^{l}}{2}} \\
\mathscr{O}\left(h_{\max }^{2^{l+1}-1}\right)\left(\lambda-q_{\max }\right)^{-\frac{2^{l}+1}{2}} \\
\mathscr{O}\left(h_{\max }^{2^{++1}-1}\right)\left(\lambda-q_{\max }\right)^{-\frac{2^{l}-1}{2}}
\end{array}\right], \text { w.r.t (12). }
\end{aligned}\right.
\end{aligned}
$$

\section{Proof of Theorem 5}

Recall the Baker-Campbell-Hausdorff-type formulas

$$
\begin{aligned}
e^{\mathbf{X}} e^{\mathbf{Y}} & =e^{\mathbf{X}+\mathbf{Y}+\frac{1}{2}[\mathbf{X}, \mathbf{Y}]+\frac{1}{12}([\mathbf{X},[\mathbf{X}, \mathbf{Y}]]+[\mathbf{Y},[\mathbf{Y}, \mathbf{X}]])+\cdots} \\
e^{\mathbf{X}} e^{\mathbf{Y}} e^{-\mathbf{X}} & =e^{\mathbf{Y}+[\mathbf{X}, \mathbf{Y}]+\frac{1}{2}[\mathbf{X},[\mathbf{X}, \mathbf{Y}]]+\frac{1}{6}[\mathbf{X},[\mathbf{X},[\mathbf{X}, \mathbf{Y}]]]+\cdots} \\
& =\exp \left(\operatorname{Ad}_{\exp (\mathbf{X})}(\mathbf{Y})\right)
\end{aligned}
$$

The local error can be written as

$$
\begin{aligned}
& \mathbf{L}_{\lambda, n}\left(c_{k}, c_{k+1}\right)= \\
& =\log \left(\mathbf{F}_{\lambda}\left(c_{k}, c_{k+1}\right) \tilde{\mathbf{F}}_{\lambda, n}^{-1}\left(c_{k}, c_{k+1}\right)\right) \\
& =\log \left(\left(\prod_{l=0}^{\infty} e^{\mathbf{D}_{\lambda, l}\left(c_{k}, c_{k+1}\right)}\right)\left(\prod_{l=0}^{n} e^{\mathbf{D}_{\lambda, l}\left(c_{k}, c_{k+1}\right)}\right)^{-1}\right) \\
& =\log \left(\left(\prod_{l=0}^{n} e^{\mathbf{D}_{\lambda, l}\left(c_{k}, c_{k+1}\right)}\right)\left(\prod_{l=n+1}^{\infty} e^{\mathbf{D}_{\lambda, l}\left(c_{k}, c_{k+1}\right)}\right)\left(\prod_{l=0}^{n} e^{\mathbf{D}_{\lambda, l}\left(c_{k}, c_{k+1}\right)}\right)^{-1}\right) \\
& =\log \left(\left(\prod_{l=0}^{n} e^{\mathbf{D}_{\lambda, l}\left(c_{k}, c_{k+1}\right)}\right) e^{\mathbf{D}_{\lambda, n+1}\left(c_{k}, c_{k+1}\right)+\text { h.o.t. }}\left(\prod_{l=0}^{n} e^{\mathbf{D}_{\lambda, l}\left(c_{k}, c_{k+1}\right)}\right)^{-1}\right) \\
& =\log \left(e^{\mathbf{D}_{\lambda, 0}\left(c_{k}, c_{k+1}\right)} e^{\mathbf{D}_{\lambda, n+1}\left(c_{k}, c_{k+1}\right)+\text { h.o.t. }} e^{-\mathbf{D}_{\lambda, 0}\left(c_{k}, c_{k+1}\right)}\right) \\
& =\operatorname{Ad}_{\exp \left(\mathbf{D}_{\lambda, 0}\left(c_{k}, c_{k+1}\right)\right)}\left(\mathbf{D}_{\lambda, n+1}\left(c_{k}, c_{k+1}\right)+\text { h.o.t. }\right) \\
& =\operatorname{Ad}_{\exp \left(\mathbf{D}_{\lambda, 0}\left(c_{k}, c_{k+1}\right)\right)}\left(\mathbf{D}_{\lambda, n+1}\left(c_{k}, c_{k+1}\right)\right)+\text { h.o.t. }
\end{aligned}
$$


where the first and second equalities are due to Definition 6 , the fourth equality is due to (38), the fifth equality is due to (39), and the sixth equality is due to 40). The local error expression (41), together with Theorem 4, yields the desired estimate.

The global error obeys the recursion relation with initial condition

$$
\mathbf{G}_{\lambda, n}\left(c_{1}\right)=\mathbf{L}_{\lambda, n}\left(a, c_{1}\right)
$$

and general rule

$$
\begin{aligned}
& \mathbf{G}_{\lambda, n}\left(c_{k+1}\right)= \\
& =\log \left(\mathbf{Y}_{\lambda}\left(c_{k+1}\right) \tilde{\mathbf{Y}}_{\lambda, n}^{-1}\left(c_{k+1}\right)\right) \\
& =\log \left(\mathbf{F}_{\lambda}\left(c_{k}, c_{k+1}\right) \mathbf{Y}_{\lambda}\left(c_{k}\right) \tilde{\mathbf{Y}}_{\lambda, n}^{-1}\left(c_{k}\right) \tilde{\mathbf{F}}_{\lambda, n}^{-1}\left(c_{k}, c_{k+1}\right)\right) \\
& =\log \left(\mathbf{F}_{\lambda}\left(c_{k}, c_{k+1}\right) e^{\mathbf{G}_{\lambda, n}\left(c_{k}\right)} \tilde{\mathbf{F}}_{\lambda, n}^{-1}\left(c_{k}, c_{k+1}\right)\right) \\
& =\log \left(e^{\mathbf{L}_{\lambda, n}\left(c_{k}, c_{k+1}\right)} \tilde{\mathbf{F}}_{\lambda, n}\left(c_{k}, c_{k+1}\right) e^{\mathbf{G}_{\lambda, n}\left(c_{k}\right)} \tilde{\mathbf{F}}_{\lambda, n}^{-1}\left(c_{k}, c_{k+1}\right)\right) \\
& =\log \left(e^{\mathbf{L}_{\lambda, n}\left(c_{k}, c_{k+1}\right)}\left(\prod_{l=0}^{n} e^{\mathbf{D}_{\lambda, l}\left(c_{k}, c_{k+1}\right)}\right) e^{\mathbf{G}_{\lambda, n}\left(c_{k}\right)}\left(\prod_{l=0}^{n} e^{\mathbf{D}_{\lambda, l}\left(c_{k}, c_{k+1}\right)}\right)\right. \\
& =\log \left(e^{-1}\right) \\
& =\log \left(e^{\mathbf{L}_{\lambda, n}\left(c_{k}, c_{k+1}\right)} e^{\mathbf{D}_{\lambda, 0}\left(c_{k}, c_{k+1}\right)} \exp \left(\operatorname{Ad}_{\exp \left(\mathbf{D}_{\lambda, 0}\right)} e^{\left.\left.\mathbf{G}_{\lambda, n}\left(c_{k}\right)+c_{k+1}\right)\right)}\left(\mathbf{G}_{\lambda, n}\left(c_{k}\right)+\text { h.o.t. }\right)\right)\right) \\
& =\log \left(e^{-\mathbf{L}_{\lambda, n}\left(c_{k, 0}, c_{k+1}\right)} \exp \left(\operatorname{Ad}_{\exp \left(\mathbf{D}_{\lambda, 0}\left(c_{k}, c_{k+1}\right)\right)}\left(\mathbf{G}_{\lambda, n}\left(c_{k}\right)\right)+\text { h.o.t. }\right)\right) \\
& =\mathbf{L}_{\lambda, n}\left(c_{k}, c_{k+1}\right)+\operatorname{Ad}_{\exp \left(\mathbf{D}_{\lambda, 0}\left(c_{k}, c_{k+1}\right)\right)}\left(\mathbf{G}_{\lambda, n}\left(c_{k}\right)\right)+\text { h.o.t. }
\end{aligned}
$$

where the first, second, third, fourth and fifth equalities are due to Definition 6, the sixth equality is due to (39), the seventh equality is due to (40), and the last equality is due to (38). The global error expressions (42) and (43) lead to

$$
\begin{aligned}
\mathbf{G}_{\lambda, n}\left(c_{k+1}\right)= & \operatorname{Ad}_{\exp \left(\mathbf{D}_{\lambda, 0}\left(c_{k}, c_{k+1}\right)\right)}\left(\mathbf{D}_{\lambda, n+1}\left(c_{k}, c_{k+1}\right)\right)+ \\
& +\operatorname{Ad}_{\exp \left(\mathbf{D}_{\lambda, 0}\left(c_{k}, c_{k+1}\right)\right) \exp \left(\mathbf{D}_{\lambda, 0}\left(c_{k-1}, c_{k}\right)\right)}\left(\mathbf{D}_{\lambda, n+1}\left(c_{k-1}, c_{k}\right)\right)+ \\
& +\cdots+ \\
& +\operatorname{Ad}_{\exp \left(\mathbf{D}_{\lambda, 0}\left(c_{k}, c_{k+1}\right)\right) \cdots \exp \left(\mathbf{D}_{\lambda, 0}\left(a, c_{1}\right)\right)}\left(\mathbf{D}_{\lambda, n+1}\left(a, c_{1}\right)\right)+ \\
& + \text { h.o.t. }
\end{aligned}
$$

which, together with 6 and Theorem 4 result in the desired estimate.

\section{Acknowledgments}

The authors would like to thank the anonymous reviewers for their valuable comments and suggestions that significantly contributed to improving the quality of the paper. The work of A. G. C. P. Ramos was supported by Fundação para a Ciência e a Tecnologia, Portugal through the fellowship SFRH/BD/71692/2010. 


\section{References}

Driscoll, T., Hale, N. and Trefethen, L., eds (2014), Chebfun Guide, Pafnuty Publications, Oxford.

Everitt, W. N. (2005), A catalogue of Sturm-Liouville differential equations, in W. O. Amrein, A. M. Hinz and D. P. Pearson, eds, 'Sturm-Liouville Theory: Past and Present', Birkhäuser Verlag Basel, pp. 271-331.

Fer, F. (1958), 'Résolution del l'equation matricielle $\dot{U}=p U$ par produit infini d'exponentielles matricielles', Bulletin de la Classe des Sciences Académie Royale de Belgique 44, 818-829.

Iserles, A. (1984), 'Solving linear ordinary differential equations by exponentials of iterated commutators', Numerische Mathematik 45(2), 183-199.

Iserles, A., Munthe-Kaas, H. Z., Nørsett, S. and Zanna, A. (2000), 'Lie-group methods', Acta Numerica 9, 215-365.

Iserles, A. and Nørsett, S. (1999), 'On the solution of linear differential equations on Lie-groups', Philosophical Transactions of the Royal Society A 357, 983-1019.

Ixaru, L. (2000), 'CP methods for the Schrödinger equation', Journal of Computational and Applied Mathematics 125(1-2), 347-357.

Ledoux, V., Daele, M. V. and Berghe, G. V. (2005), 'Matslise: A matlab package for the numerical solution of sturm-liouville and Schrödinger equations', ACM Transactions on Mathematical Software 31(4), 532-554.

Ledoux, V., Daele, M. and van den Berghe, G. (2010), 'Efficient numerical solution of the 1D Schrödinger eigenvalue problem using magnus integrators', IMA Journal of Numerical Analysis 30, 751-776.

Moan, P. C. (1998), Efficient approximation of Sturm-Liouville problems using Liegroup methods, Technical report, Department of Applied Mathematics and Theoretical Physics, University of Cambridge, United Kingdom.

Pryce, J. (1993), Numerical Solution of Sturm-Liouville Problems, Oxford University Press.

Zanna, A. (1996), The method of iterated commutators for ordinary differential quations on Lie groups, Technical report, Department of Applied Mathematics and Theoretical Physics, University of Cambridge, United Kingdom.

Zanna, A. (1998), On the Numerical Solution of Isospectral Flows, PhD thesis, University of Cambridge, United Kingdom.

Zettl, A. (2005), Sturm-Liouville Theory, American Mathematical Society. 